\title{
What have genome-wide association studies taught us about cGMP and blood pressure regulation?
}

\author{
Christopher Newton-Cheh ${ }^{1,2}$ \\ From 6th International Conference on cGMP: Generators, Effectors and Therapeutic Implications \\ Erfurt, Germany. 28-30 June 2013
}

\section{Background}

Hypertension (HTN) is a major worldwide cause of stroke, heart failure, myocardial infarction, and chronic kidney disease. Mechanisms of blood pressure regulation have previously been elucidated from the study of animal models, Mendelian hyper- and hypo-tension in humans, and drug treatment trials. More recently, genome-wide association studies (GWASs) have offered unbiased approaches to identify novel mechanisms contributing to blood pressure control. We and others have reported candidate gene and GWASs that have identified common genetic variants of modest effect on blood pressure that fall in pathways involving natriuretic peptide and nitric oxide signalling [1-3].

\section{Results}

Common variant rs5068 in the 3' UTR of NPPA, which encodes the atrial natriuretic peptide (ANP), is associated with higher plasma ANP and lower blood pressure and odds of hypertension [2]. We have now completed a genotype-directed physiologic study of 8 heterozygotes and 23 major homozygotes demonstrating that the variant alters the "set point" of ANP $(\mathrm{p}=0.02)$ but does not alter the ANP response to high vs low sodium diet $(\mathrm{p}<0.0001)$ or to intravenous saline infusion ( $\mathrm{p}<0.0001$, in press); additional evidence for the mechanism by which this variant acts will be presented in a separate abstract. Downstream of the natriuretic peptide system, a common noncoding variant at a locus including NPR3, encoding the natriuretic peptide clearance receptor, has been associated with blood pressure although it is not associated with plasma ANP or BNP [3]. A common variant at the locus including ENOS,

Correspondence: cnewtoncheh@chgr.mgh.harvard.edu

${ }^{1}$ Massachusetts General Hospital, Boston, MA, USA

Full list of author information is available at the end of the article encoding endothelial nitric oxide synthase, is associated with blood pressure and hypertension [4,5]. Lastly, an intronic variant in GUCY1A3, encoding the alpha subunit of soluble guanylate cyclase, is associated with blood pressure and myocardial infarction risk.

\section{Conclusion}

The natriuretic peptide and nitric oxide systems are important contributors to blood pressure regulation in humans and represent strong targets for pharmacologic intervention to reduce the burden of disease from hypertension.

\section{Authors' details}

${ }^{1}$ Massachusetts General Hospital, Boston, MA, USA. ${ }^{2}$ Broad Institute of Harvard and MIT, Cambridge, MA, USA.

Published: 29 August 2013

\section{References}

1. Newton-Cheh C, et al: Genome-wide association study identifies eight loc associated with blood pressure. Nat Genet 2009, 41:666-676.

2. Newton-Cheh $C$, et al: Association of common variants in NPPA and NPPB with circulating natriuretic peptides and blood pressure. Nat Genet 2009, 41:348-353.

3. International Consortium for Blood Pressure Genome-Wide Association: Genetic variants in novel pathways influence blood pressure and cardiovascular disease risk. Nature 2011, 478:103-109.

4. Johnson T, et al: Blood Pressure Loci Identified with a Gene-Centric Array. The American Journal of Human Genetics 2011, 89:1-13.

5. Salvi $E$, et al: Genomewide association study using a high-density single nucleotide polymorphism array and case-control design identifies a novel essential hypertension susceptibility locus in the promoter region of endothelial NO synthase. Hypertension 2012, 59:248-255.

doi:10.1186/2050-6511-14-S1-O5

Cite this article as: Newton-Cheh: What have genome-wide association studies taught us about cGMP and blood pressure regulation? BMC Pharmacology and Toxicology 2013 14(Suppl 1):O5. 